\title{
Development of a friction sensor for hot forging
}

\author{
Takeshi Yoneyama ${ }^{1,2}$
}

Received: 8 August 2016 / Accepted: 25 September 2016 /Published online: 12 October 2016

(C) The Author(s) 2016. This article is published with open access at Springerlink.com

\begin{abstract}
A friction sensor that can detect both pressure and frictional stress on the tool's surface during hot forging has been developed. The detection principle was introduced in 2004 by the present author; however, a sensor suitable to be installed in the forging die has not been created until now. In this study, a hot forging sensor prototype is presented, and its design method is described in detail. The sensor comprises two beams connected to the thin plate part of the top surface of the sensor pin, and a thin plate connects the base of the two beams. According to the friction applied on the sensor surface, the two beams incline to deform the thin plate at the base of the beams. The frictional stress on the sensor surface corresponds to the difference between the two strains on the thin plate surface. The main dimensions of the sensor structure are determined according to the expected applied pressure and frictional stress. Additionally, a new calibration test equipment is developed to obtain the calibration coefficient. Finally, the pressure and the frictional stress are measured in hot forging, as illustrated in an example, and the validity of the output of the friction sensor is confirmed.
\end{abstract}

Keywords Friction $\cdot$ Contacting stress $\cdot$ Forging $\cdot$ Sensor . Pressure $\cdot$ Tool $\cdot$ Measurement

Takeshi Yoneyama

yoneyama@se.kanazawa-u.ac.jp

Kanazawa University, Kakuma-machi, Kanazawa 920-1192, Japan

2 School of Mechanical Engineering, Kanazawa University, Kakuma-machi, Kanazawa 920-1192, Japan

\section{Introduction}

The stress boundary conditions at the work interface and the tool have significant effects on the plastic deformation of the work material. Detecting contact stress on the tool surface is important not only to understand the boundary phenomenon during the plastic deformation process but also to provide the appropriate boundary conditions for the numerical analysis of the metal-forming process. Siebel and Lueg [1] first developed the pressure pin technique in order to detect the contacting pressure. MacGregor and Palme [2] measured the distribution of the contacting pressure in the rolling of metals. Matsuura and Motomura [3] investigated the distribution of rolling pressure by using the pressure pin technique. Further, Motomura [4] studied the metal hot rolling pressure distribution. Kato et al. [5] developed a multichannel system capable of measuring the rolling pressure. Tozawa et al. [6] investigated the conditions of measuring the rolling pressure by using the pin method. Ishikawa et al. [7] measured the pressure distribution under various rolling conditions.

Van Rooyen and Bachofen [8] applied the pressure pin technique in order to detect frictional stress in cold rolling as the oblique pin mechanism. Matsuura and Motomura [9] investigated the variation of the friction coefficient along the contact arc and the length of the arc by using the oblique pin mechanism. Watanabe [10] studied the influences of strip tensions on the distributions of roll pressure, tangential shear stress, and friction coefficient in the roll gap by using the same mechanism.

Yoneyama and Hatamura [11-12] developed a stress sensor, which can detect both the pressure and the frictional stress in any direction by a single detecting pin by using a threedirectional force-detecting structure. Yoneyama [13] presented several examples of measuring the pressure and frictional stress by using this sensor. Jeswiet and Nyahumwa [14] also 
developed a sensor that can detect both the pressure and the frictional stress. Nyahumwa [15] applied this technique to detect the frictional forces during cold rolling. However, these pressure pin techniques have a gap between the detection pin and the surrounding hole in the tool, where deformed material may flow into the gap. In addition, keeping the same surface level between the sensor and tool surfaces is difficult during the metal-forming process and its influence on the friction condition.

To eliminate the gap and the level difference in the measurement of contacting stress, Yoneyama and Hatamura [16] developed a pressure sensor by using an optical fiber displacement meter. Yoneyama et al. [17] applied this method to the measurement of contacting stress in extrusion. Dellah et al. [18] developed an embedded strain-gauged diaphragm sensor press fit on the tool surface. Lupoi and Osman [19] developed under surface pressure sensing technique which was explored from the pressure pin technique. Jeswiet et al. [20] presented sealed cantilever pin for the measurement of friction in a rolling process.

Yoneyama [21] developed a pressure sensor by using strain gauges with a structure comprising an inner shaft and an outer tube connected at the tool surface and combined with a thin plate at the both ends. Subsequently, Yoneyama started developing a sensor to detect both the pressure and the frictional stress without a gap and a level difference between the sensor and the tool surface. Yoneyama and Takahashi [22-23] proposed an appropriate structure, and the developed sensor was applied to detect the pressure and the friction on a container surface in an aluminum hot extrusion. Makino et al. [24] developed a pressure and friction sensor with the center pole under the thin plate of the tool.

In the field of forging, there is no attempt except ours to detect pressure and friction on the surface of tools during the actual metal-forming process. The friction condition is usually investigated through various tests, including ring compression, double-cup extrusion, spike test, and T-shape test [25]. These tests evaluate the friction condition by the comparison of total load and deformation with numerical analysis by using almost constant friction condition on the tool surface. However, in order to elucidate real friction acting on the tool surface during forging, the pressure and frictional stress on the tool surface need to be measured directly.

Shimoda and Yoneyama [26] detected the pressure and the friction on the flash land surface in the hot forging of the crankshaft. However, the details of that sensor design were not presented.

In the present study, a prototype of a friction sensor is presented, and its design method is investigated in detail. In addition, the calibration test equipment is designed and investigated. Finally, a measurement example in hot forging is shown to illustrate the applicability of this sensor to various metal-forming processes.

\section{Design and fabrication of friction sensor}

\subsection{Structural design}

At first, the problems of the pressure pin-type sensors and sensors developed by other researchers are described by Fig. 1. Problems in pressure pin-type sensor are illustrated in Fig. 1a-c. Due to the difference between the elastic compliance of the sensor and that of the surrounding tool, a level gap occurs between the top surface of the sensor and the surrounding hole edge corner on the tool's surface. The depression in the top surface of the sensor, as shown in Fig. 1a, causes less pressure to be applied on the sensor than to the tool's surface. Conversely, a protrusion, as shown in Fig. 1b, causes more pressure to be applied on the sensor's surface than on to the tool's surface. Both of these scenarios cause resistance to the flow of material on the tool's surface. Material often enters the peripheral space between the detection pin and the insertion hole during plastic deformation, as shown in Fig. 1c. This leads to the sensor malfunction and resistance to the flow of material.

An embedded strain-gauged diaphragm sensor, developed by Dellah et al. as shown in Fig. 1d, provides a good way to detect the friction acting on a tool's surface; it does this through the differences in strain on the rear surface of the diaphragm. This structure is too weak to measure high pressure in the usual forging process, and it seems to have an interference problem. Moreover, pressure applied on the surrounding tool surface affects the deformation of the press-fit diaphragm even if there is no material on the diaphragm's surface. Under surface pressure sensing technique, developed by Lupoi and Osman as shown in Fig. 1e, resolves the level difference between the pin and the edge of the hole. Nevertheless, it still needs two sensors, in combination with a perpendicular pin and an oblique pin, in order to obtain frictional stress. It also has an interference problem that relates to the deformation of the surrounding tool surface on the detection of pressure pin. A sealed cantilever pin proposed by Jeswiet et al. and illustrated in Fig. 1f resolves the problem of the material that falls between the pin and the hole. Its frictionsensing performance seems to be reduced, and it also has an interference problem relating to the deformation of the surrounding tool surface on the output of the sensor pin.

The principle for detecting pressure and friction of the sensor presented by the author is shown in Fig. 2. The sensor comprises a thin plate on the tool surface, two beams connected on the rear surface of the thin plate, and another thin plate, which connects the two beams at the base. This structure is usually machined from a single block via electrical discharge machining. When pressure is applied on the tool surface, the thin plate part of the tool surface deforms as illustrated in Fig. 2a. The two beams are deformed. Finally, the thin plate at the bottom deforms causing the surface strain. Strain gauges 
Fig. 1 Problems of pressure pintype sensor and solutions by other researchers

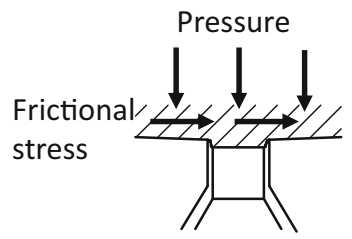

(a) Detecting pin sinks under the tool surface

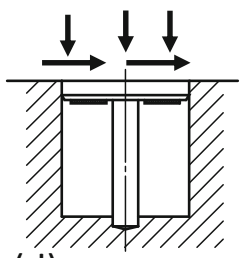

(d) Embedded diaphragm sensor (Dellah et al.)

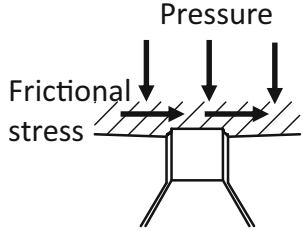

(b) Detecting pin protrudes over the tool surface

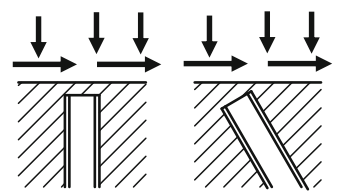

(e) Under surface pressure sensing technique (Lupoi \& Osman)

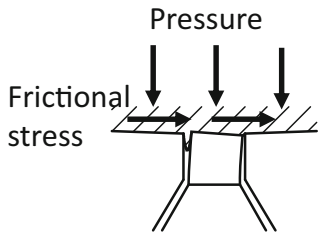

(c) Material extrudes into the gap around the sensing pin

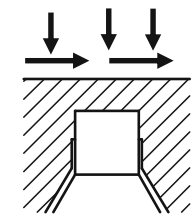

(f) Sealed cantilever pin (Jeswiet et al.) are fit at both ends of the bottom thin plate. The total pressure can be calculated from the sum of the two strains. When friction is applied on the tool surface, one side of the thin plate, at the beginning of the friction vector, bends downward. On the other hand, the ending side of the friction vector bends upward. From the difference in the bending directions, the end positions of the two beams shift. Consequently, the thin plate at the bottom is deformed, as shown in Fig. $2 b$ with different strains on each side. The friction on the tool surface corresponds to the difference between the two strains.

To apply the above structural principles to the measurement in hot forging, the sensor structure illustrated in Fig. 3 has been designed. To measure the pressure and frictional stress on the tool surface, which has various profiles, a pin-type sensor that can be inserted on a hole machined on a tool surface has an advantage. In particular, the machining of the tool and the manufacturing of the sensor are separated. Thus, the manufacturing of the sensor is easier than if it was directly made on the tool. One of the problems in machining the sensor structure is to create a square hole surrounded by a thin plate tool surface, two beams, and a bottom thin plate in the sensor pin. To manufacture this sensor structure from a single pin block, a space is necessary, where an electrode of electrical discharge machining will be inserted. This is because the
Fig. 2 The principle of the sensor structure for detecting pressure and friction [14]

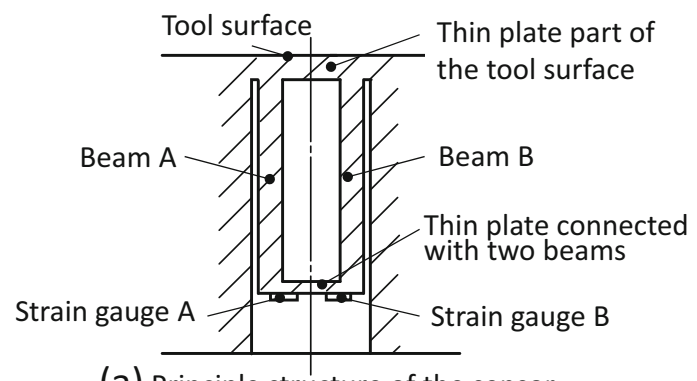

(a) Principle structure of the sensor

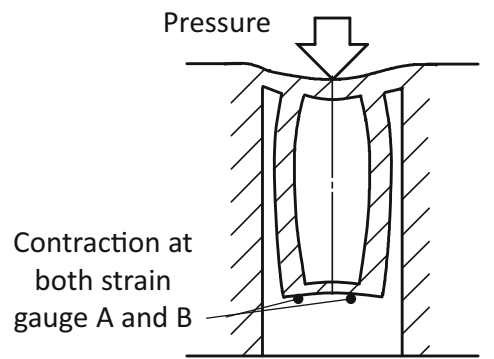

(b) Deformation by pressure

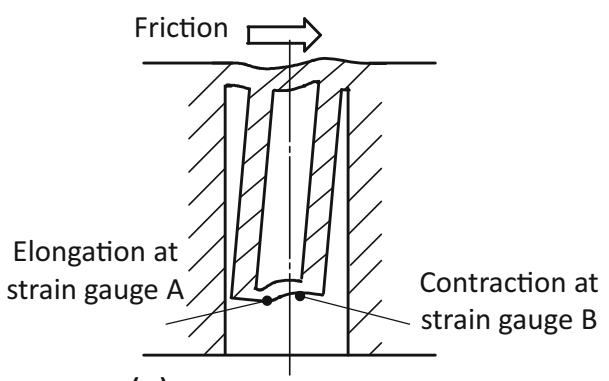

(c) Deformation by friction 


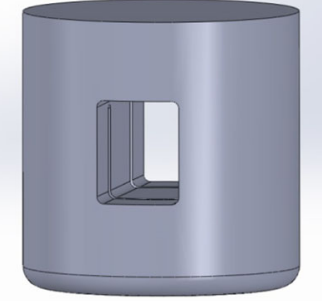

(a) Isometric view of the sensor model

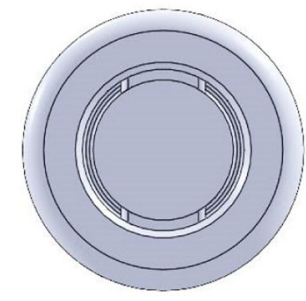

(c) Bottom view of the sensor pin

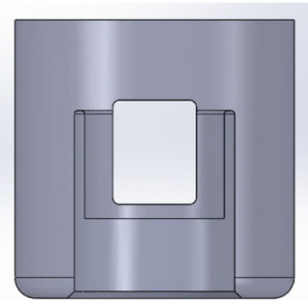

(b) Cross section at the center plane of the sensor pin

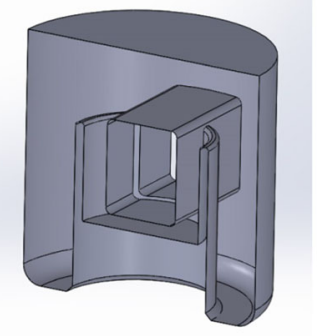

(d) Cutting space in the circular pin
Fig. 3 Designed sensor pin structure

detecting block is surrounded by the circular tube wall of the sensor block. The solution in this design is to create a square hole outside the pin through the circular wall of the sensor pin. Using this method, the machining process of the sensor pin becomes simple, because this process comprises only two steps. The first step is to make a circular hole and a cylindrical concavity to form a cylindrical block connected to the rear side of the thin plate part of the top surface. The second step is to create a side square hole to let the thin plate part connected at the bottoms of the two beams. This sensor is called as "friction sensor" in this study.

\subsection{Sensor pin dimensions}

To apply the friction sensor to hot forging, the diameter and the height of a sensor pin are set at $8 \mathrm{~mm}$ each. In the sensor design, the dimensions shown in Fig. 4 must be decided

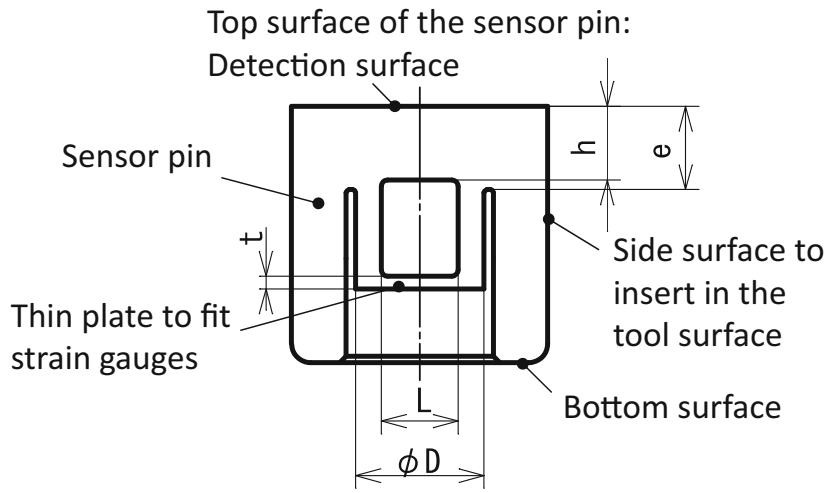

Fig. 4 Dimensions to be decided according to the condition of the expected pressure and friction. To determine the appropriate dimensions, a simple simulation of the deformation and strain is performed. In the finite element analysis, the fixing condition must be considered. The bottom surface of the sensor pin is rigidly fixed. On the other hand, the outer cylindrical surface of the sensor pin should not be rigidly fixed because the whole pin is compressed downward by the pressure applied on the top surface. As the friction is applied on the top surface, the sensor pin is deformed toward the friction direction. According to the tool bulk around the sensor pin, the deformation of the pin in this direction must be restricted. However, the way in which the deformation is restricted is unclear because the surrounding tool bulk is deformed by friction applied on the tool surface. In this simulation, the outer cylindrical surface is not assigned any fixing condition. The purpose of this simulation is to roughly estimate and confirm the tendency of the strain on the thin plate surface to fit the strain gauges and not to analyze the precise qualitative strain value.

An example of the deformation of the sensor structure by applying a pressure of $500 \mathrm{MPa}$ and the strain induced on the thin plate surface to fit strain gauges is shown in Fig. 5. It is confirmed that the contraction strain is induced along the $\mathrm{X}$ direction.

An example of the deformation of the sensor structure by only a $30 \mathrm{MPa}$ friction and the strain induced on the detection surface to fit strain gauges is shown in Fig. 6. Elongation occurs on the left side of the thin plate surface, whereas contraction occurs on the right side. It is confirmed that the opposite strains are produced by the application of frictional stress on the top surface.

Based on these results, the sensor dimensions were determined, as shown in Fig. 7a, and the sensor pins were machined, as shown in Fig. 7b.

\subsection{Fabrication of a friction sensor}

As the strain gauges to fit on the bottom thin plate in the sensor, a small strain gauge, KFH-02-120-C11-11H4M3, produced by Kyowa Electronic Instruments Co. Ltd., was selected. The upper limit of the operating temperature of the strain gauge is at $250{ }^{\circ} \mathrm{C}$. The required one is above $200{ }^{\circ} \mathrm{C}$ because the usual tools are heated around $200{ }^{\circ} \mathrm{C}$ before hot forging. The dimensions of the strain gauge base are $2.2 \mathrm{~mm}$ in width and $2.5 \mathrm{~mm}$ in length. The gauge length is $0.2 \mathrm{~mm}$. There is another strain gauge that has $350{ }^{\circ} \mathrm{C}$ limit of the operating temperature. However, its size is too large to fit on the designed sensor. As the space to fit strain gauges on the bottom thin plate in the sensor pin is approximately $4 \mathrm{~mm}$ wide and $3 \mathrm{~mm}$ long for two strain gauges, the periphery of the strain gauge base was cut out to fit the detecting part of the strain gauge to the detecting position in the thin plate surface. The fitting condition of the strain gauges and the fabricated sensor 
Fig. 5 Deformation of the sensor structure by pressure and strain induced on the thin plate surface to fit strain gauges

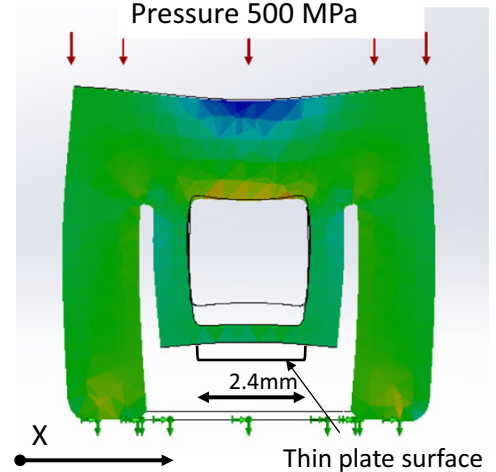

(a) Deformation by pressure

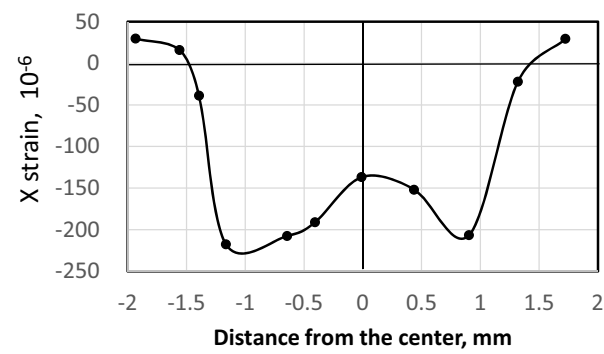

(b) Strain on the thin plate surface are shown in Fig. 8. Each strain gauge has three lead wires to cancel the changes in the electric resistance of the lead wire in case of detection by a single strain gauge. The lead wires from the strain gauge were embedded by silicon rubber and fixed in the concavity of the sensor pin, as shown in Fig. 8c.

\section{Calibration of the output of friction sensor}

\subsection{Design of calibration test equipment}

To investigate the output performance of the friction sensor and obtain the calibration coefficient, calibration test equipment is designed (Fig. 9). This equipment has one part that applies pressure on the top surface of the sensor and another part that applies friction on the same surface under pressure. A vertical load is applied through a vertical screw mechanism, and a two-dimensional load cell is attached to the metal disk holder. A horizontal force is applied through a horizontal screw mechanism, and a tensile load cell is attached to the metal disk holder. A small circular metal disk (diameter $=14 \mathrm{~mm}$ ) is used as a contacting material to apply pressure and friction. The details of the contact between the sensor surface and the metal disk are shown in Fig. 10. The applied pressure is obtained from the load divided by the contacting area of the disk. The frictional stress is the applied friction divided by the contacting area of the disk. The applied friction on the sensor is obtained from the difference between the horizontal force by the beam and the applied force on the upper contacting surface between the metal disk holder and the upper plunger because friction also occurs on this surface. For this purpose, the upper plunger has a two-dimensional load cell to obtain the vertical and tangential forces.

\subsection{Calibration results}

An example of the friction sensor's calibration output by using the calibration test equipment is shown in Fig. 11. A pure aluminum circular disk (diameter $=14 \mathrm{~mm}$ ) is used as a contacting material. As the applied pressure on the sensor surface increases, both contract strain outputs of strain gauges (1) and (2) increase. After the pressure reaches a certain predetermined value, the pressure application is stopped. Subsequently, the frictional force is applied in the horizontal direction. As the frictional stress increases, the applied pressure slightly decreases owing to some change in the contacting conditions between the
Fig. 6 Deformation of sensor structure by friction and strain induced on the thin plate surface to fit strain gauges

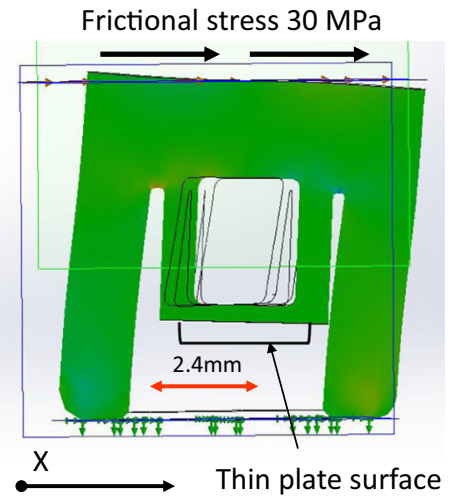

(a) Deformation by friction

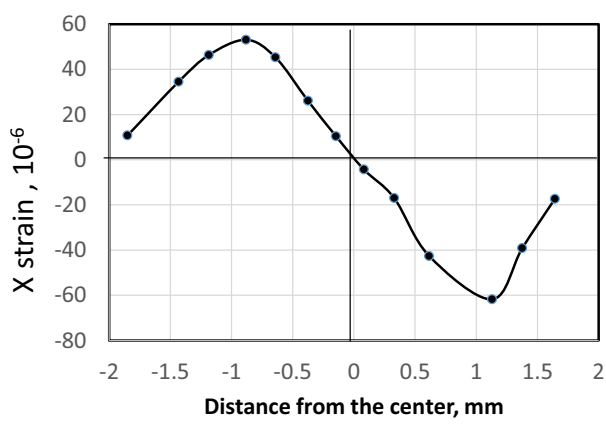

(b) Strain on the thin plate surface 


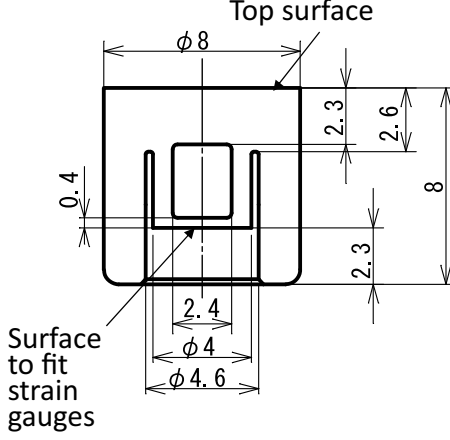

(a) Decided dimensions
Surface to fit strain gauges

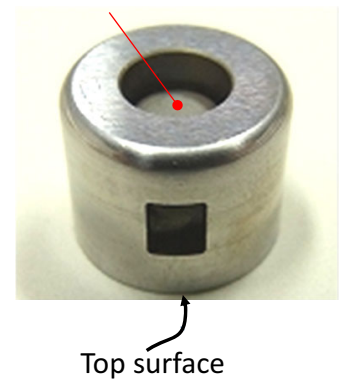

(b) Machined sensor pin
Fig. 7 Decided dimensions and machined sensor pin

aluminum disk and sensor surfaces. The output of the two strain gauges due to pressure should decrease along with the pressure. Under this situation, the output of strain gauge (1) moves to the direction of the elongation strain caused by the frictional stress. On the other hand, the output of the strain gauge (2) moves to contract the friction strain unless the contract strain decreases with the output by pressure. These changes in the two strain gauges show the output by frictional stress on the top surface. The results of the strain gauge outputs by pressure and those by friction are shown in Fig. 12. The strain outputs for pressure (a) are obtained from the first increase in the calibration test, whereas those for frictional stress (b) are obtained from the output of the strain gauges reduced with respect to the output by the pressure estimated from the relation in (a).

The two outputs of the strain gauges $O_{1}$ and $O_{2}$ can be expressed as linear functions of the pressure $p$ and the frictional stress $f$, respectively, by the following equations. In the first step of the calibration test, the outputs of the two strain gauges provide the coefficients $\alpha_{1}$ and $\alpha_{2}$, when pressure is applied. From the output changes by the application of frictional stress, the coefficients $\beta_{1}$ and $\beta_{2}$ are obtained by reducing the output by pressure by using the coefficients $\alpha_{1}$ and $\alpha_{2}$.

$$
\left.\begin{array}{l}
0_{1}=\mathrm{a}_{1} p+\beta_{1} f \\
0_{2}=\mathrm{a}_{2} p+\beta_{2} f
\end{array}\right\}
$$

The validity of the calibration equipment must be considered before using these calibration coefficients. The contacting pressure between the circular disk and the top surface of the sensor must not be uniform at the contacting surface under no lubrication. The pressure must be high at the center of the disk, and it reduces as per the distance from the center. Therefore, the applied pressure refers to the average pressure applied on the sensor surface. If the contacting material is soft, like rubber, the pressure distribution becomes uniform. Then, only a small friction could be applied owing to the slip between the rubber and sensor surfaces. In the actual forging experiment described in the next section, the pressure outputs are compared with the average actual pressure at the contact with high-temperature steel of circular disk in which the ratio between the height and the diameter is about 1.5. In that case, contacting pressure is estimated to be almost uniform during a small compression ratio. The result shows that the coefficients $\alpha_{1}$ and $\alpha_{2}$ obtained from the calibration test by using a pure aluminum disk agree well with the average contacting pressure.
Fig. 8 Strain gauge fitting and fabrication

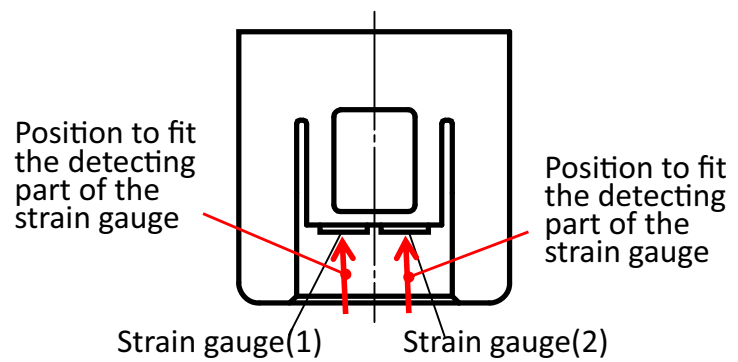

(a) Sensor structure

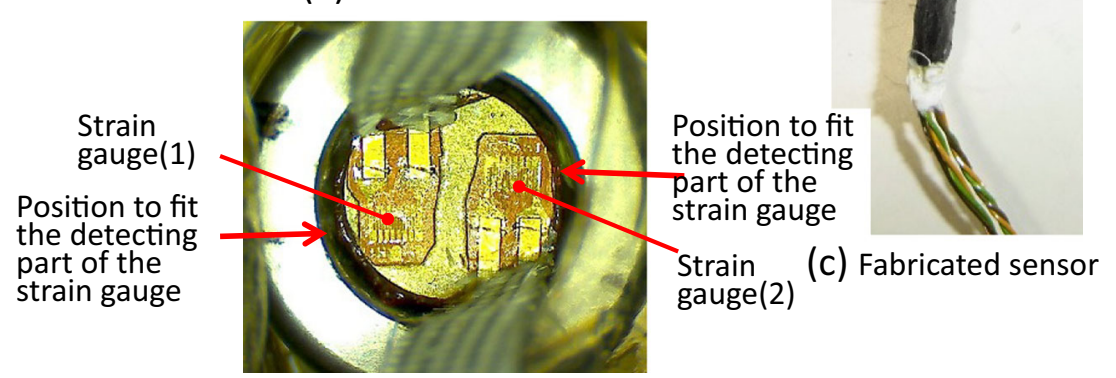

(b) Fitting of strain gauges

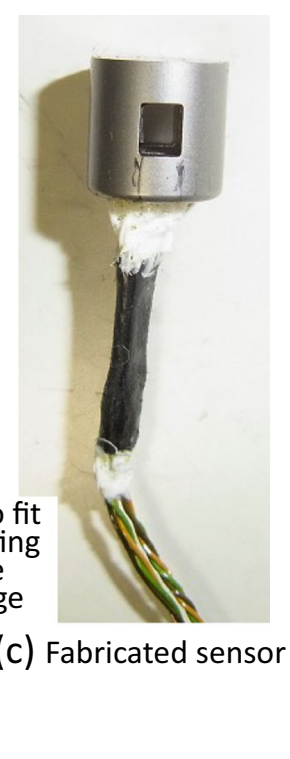

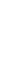


Fig. 9 Calibration test equipment

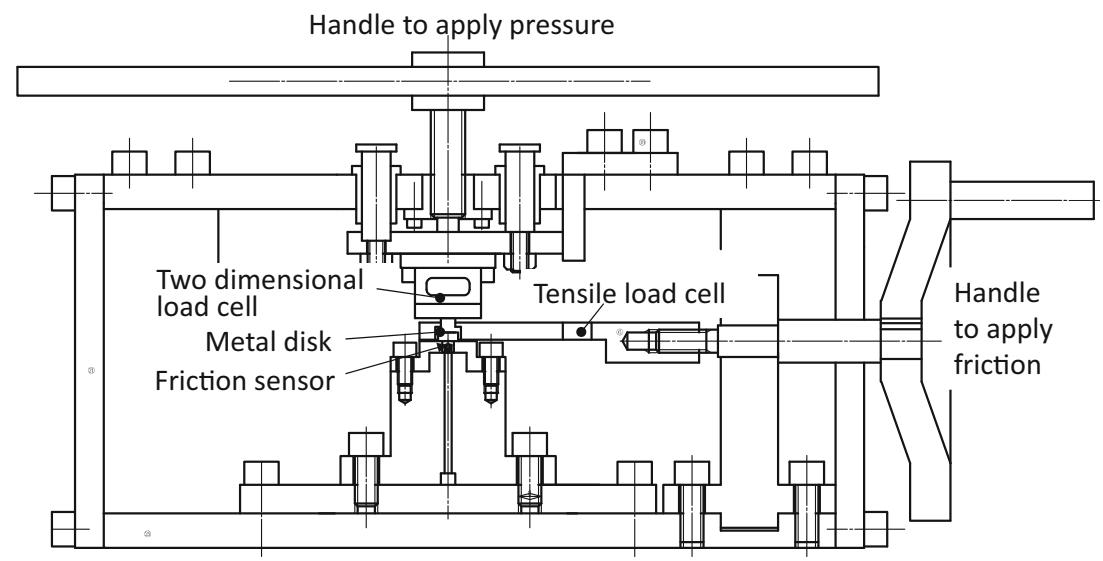

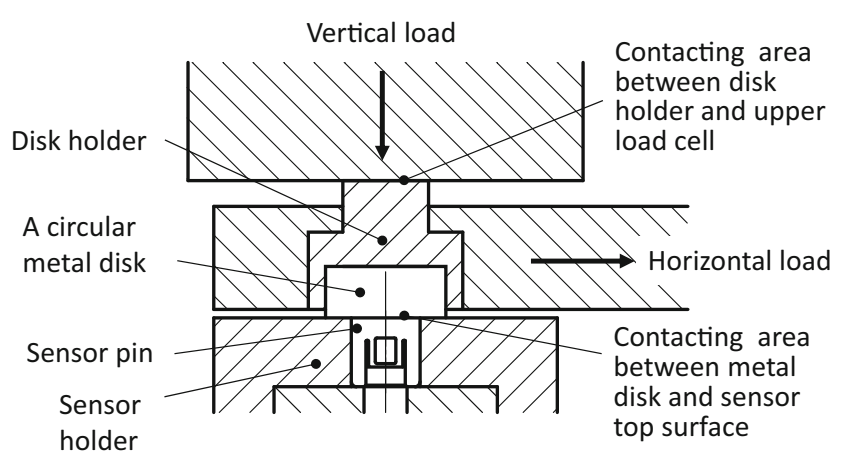

Fig. 10 Detail of the contact between the metal disk and the sensor pin in the calibration test equipment

\section{Application to the measurement in hot forging}

The proposed friction sensor was applied in the hot forging of steel. The pressure and frictional stress outputs in the upsetting of a high-temperature circular disk by using a drop hammer are shown in Fig. 13. In this process, a circular disk (diameter $=100 \mathrm{~mm}$ and height $=150 \mathrm{~mm}$ ) was compressed. First, the height was reduced from 150 to $88 \mathrm{~mm}$. Second, the height was compressed from 88 to $50 \mathrm{~mm}$. One sensor was embedded at $10 \mathrm{~mm}$ from the center in the flat lower die. Another sensor was embedded at $35 \mathrm{~mm}$ from the center. The compression stroke was obtained from an image captured by a high-speed camera. The contacting time was approximately $10 \mathrm{~ms}$. Based on the stroke and the contacting time, the average drop speed was approximately $4 \mathrm{~m} / \mathrm{s}$.

Pressure 1, $10 \mathrm{~mm}$ from the center, was higher than pressure 2, $35 \mathrm{~mm}$ from the center. This is a popular image of the compression of a circular disk as "friction hill." On the contrary, friction 2, $35 \mathrm{~mm}$ from the center, was larger than friction $1,10 \mathrm{~mm}$ from the center. The fact that the frictional stress increases as per the distance from the center during the compression of a circular disk agrees with the result that has been reported by using a pressure pin-type friction sensor [13, 19]. The total load was estimated from the pressure, and it agreed with the punch load measured in the hammer forging.

A comparison of the measured pressure and frictional stress at the final position in the forging with the pressure distribution provided by an elemental method of analysis is shown in Fig. 14. Two equations on contacting pressure $p$ as a function of the distance from the center $r$ are as follows:

$$
\begin{aligned}
& p=Y \exp \frac{2 \mu}{h}\left(r-\frac{d}{2}\right) \\
& p=\left(Y+\frac{d}{h} f\right)-\frac{2 f}{h} r
\end{aligned}
$$

Fig. 11 Applied pressure and friction and outputs of strains in the calibration

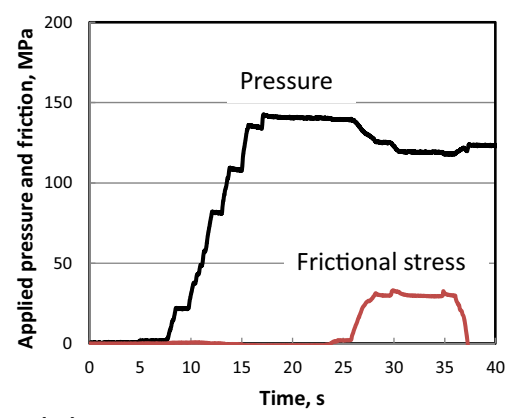

(a) Applied pressure and frictional stress

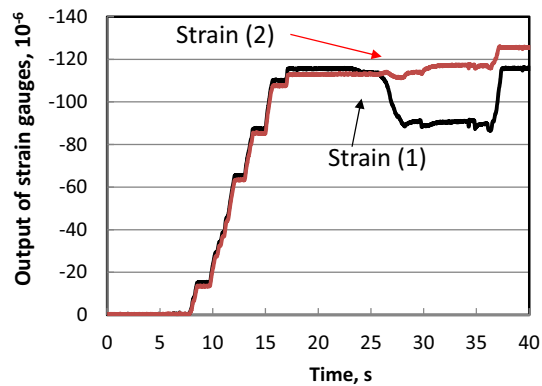

(b) Strain outputs of two strain gauges 
Fig. 12 Outputs of strains according to pressure and frictional stress

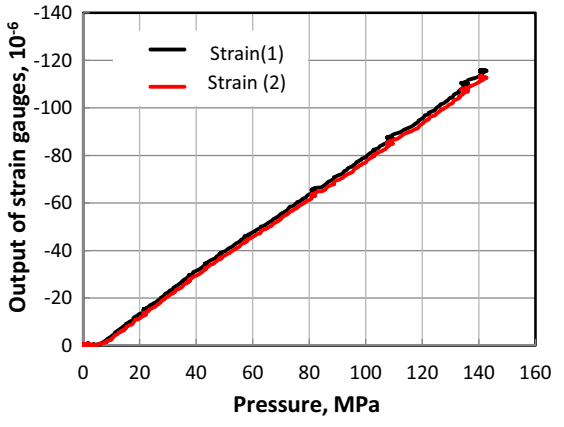

(a) Strain outputs for pressure

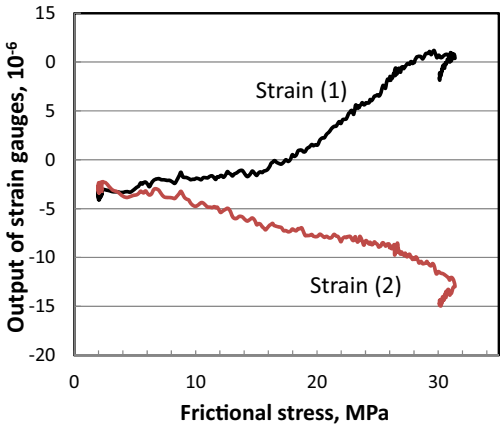

(b) Strain outputs for friction
Fig. 13 Measurement of pressure and frictional stress in the upsetting of a circular disk by drop hammer forging

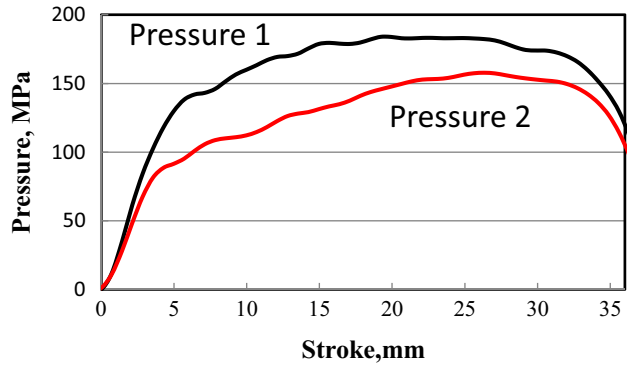

(a) Measured pressure

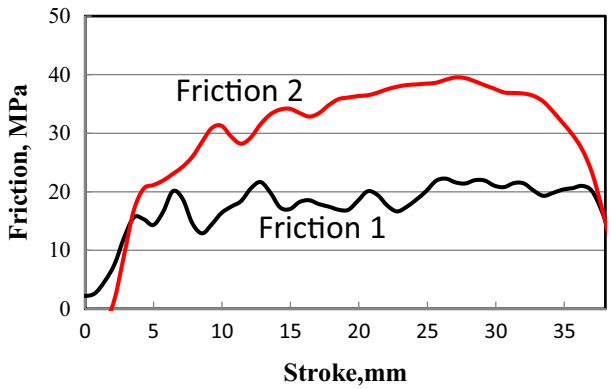

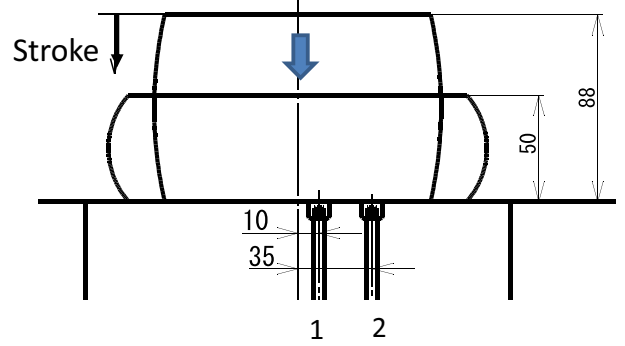

(c) Positions of sensors

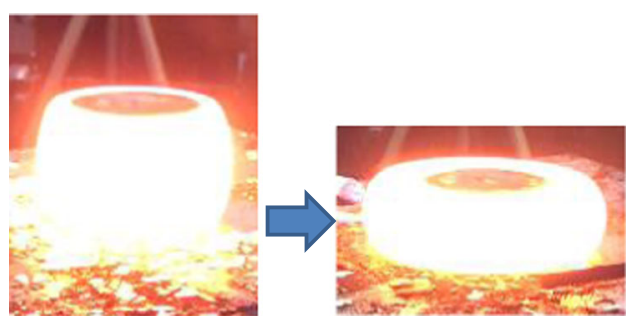

(d) Pictures of the forged steel (b) Measured frictional stress
Equation (2) is obtained on constant, where $\mu$ is the ratio of friction to pressure, $Y$ is the deformation resistance, $h$ is the height of the cylinder, and $d$ is the diameter of the circular disk. Equation (3) is obtained on uniform friction, where $f$ is frictional stress on the contacting surface.
Two analysis results appear in the figure. One is obtained by using a friction coefficient $\mu=0.3$, and the other is obtained with a uniform frictional stress of $40 \mathrm{MPa}$ from the center to the periphery. In this analysis, the deformation resistance of the forged steel $Y$ is set to $80 \mathrm{MPa}$. The measured pressures are
Fig. 14 Comparison of measured pressure and friction with elemental method of analysis at the final position

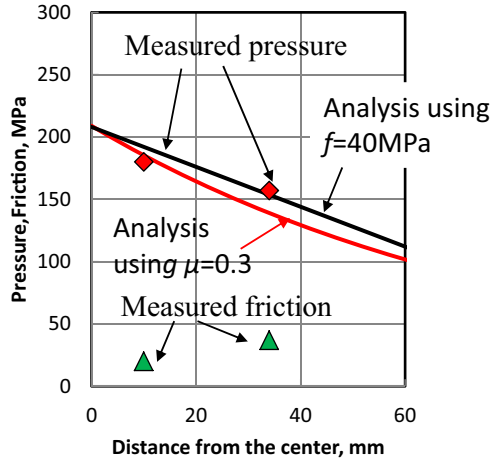

(a) Comparison of measured data with the analysis of elemental method

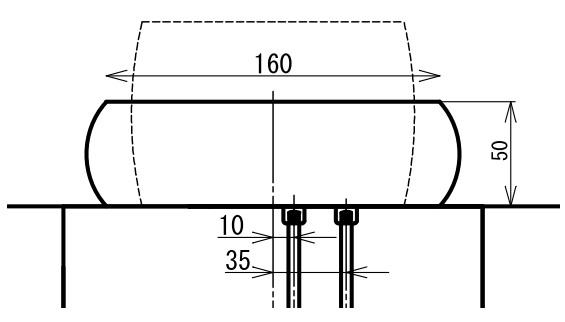

(b) Final deformation shape and contact with sensors 


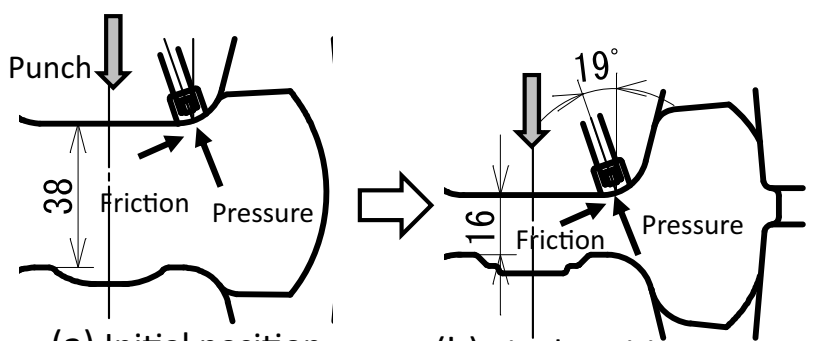

(a) Initial position

(b) Final position

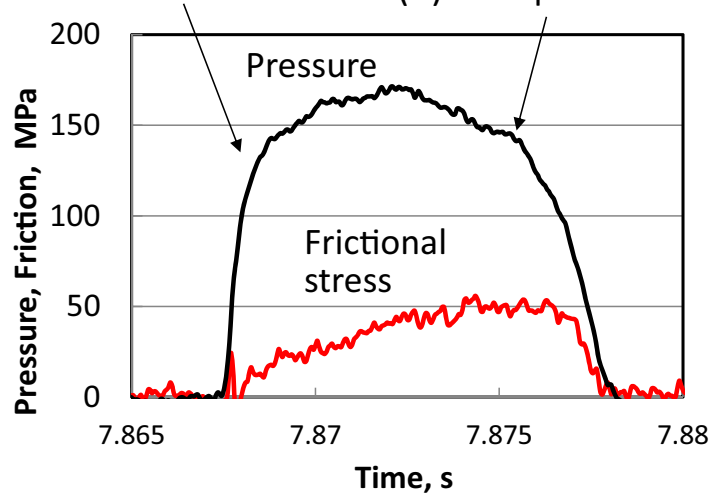

(c) Pressure and friction acting on the punch corner

Fig. 15 Pressure and frictional stress acting on the punch corner during the hot forging

nearly situated in the line of pressure analyzed with the uniform frictional stress $(40 \mathrm{MPa})$ that agrees with the measured friction. The friction coefficient $\mu=0.3$ indicates that the frictional stress near the center is approximately $60 \mathrm{MPa}$ and that at $60 \mathrm{~mm}$ is $30 \mathrm{MPa}$ depending on the pressure, which does not agree with the measured friction. In the area near the center, there is a dead zone where the material is only pressed at high pressure and does not deform to any significant degree. Around such an area, friction is not high even when the pressure is high. Therefore, friction here does not conform to the constant friction coefficient law, which dictates that an increase in pressure results in an increase in friction. As the distance from the center increases, the flow of material above the tool surface increases, and the top part of the asperity of the material that contacts with the surface of the tool deforms in microscale. This causes an increase in the real contact area between the material and tool surfaces. The friction must reach to the shear yield stress of the material because the connection between the surface of the material and that of the tool reaches to the fixed condition. Therefore, frictional stress on the tool surface is nearly constant from some distance from the center to the outside edge of the material.

This simple result demonstrates a validity of this friction sensor to investigate contacting pressure and friction on the tool surface directly during hot forging.

Another example of a sensor that can detect pressure and frictional stress in hot forging is shown in Fig. 15. A hightemperature axisymmetric steel material is compressed by an upper punch from the height shown in Fig. 15a to the height shown in Fig. 15b. In this process, outside surface of the material comes in contact with the inside circular surfaces of the upper and lower tools during the process of drop hammer forging. Pressure and frictional stress acting on the corner of the upper punch surface whose normal direction is inclined at $19^{\circ}$ to the axis of the punch were detected, as can be seen in Fig. $15 \mathrm{c}$. Although the pressure acting on the surface did not change in any significant way, frictional stress increased during the process. This simple result indicates that measurement at various positions on the tool needs to be made during the hot forging process in order to elucidate how the friction condition relates to that of material deformation, strain rate, temperature, surface roughness, and the condition of the lubricant.
Fig. 16 Image of twodimensional friction sensor

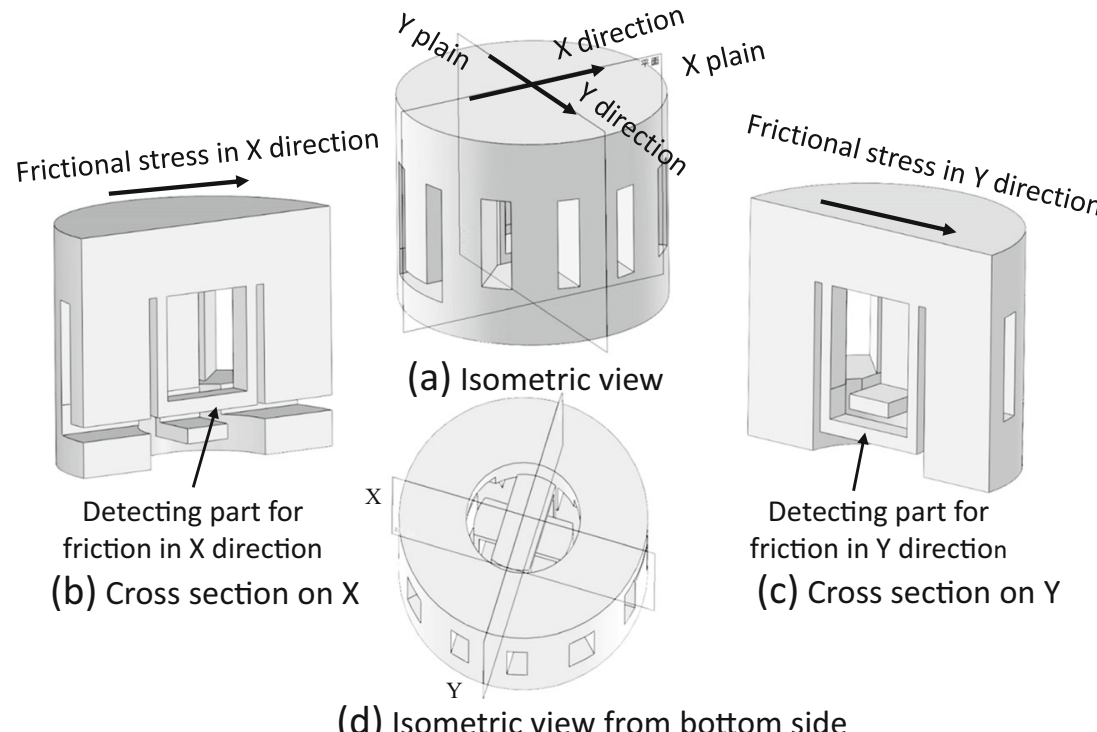

(d) Isometric view from bottom side 


\section{Discussion}

There are several points to be considered in order to apply the friction sensor to actual forging. First, it is necessary to investigate the influence of the temperature on the sensor by heat transfer from the work surface to the tool during forging. Although the zero level of the strain output changes with the temperature, there is hardly any change during the contact with the work surface because of the contacting time as short as $10 \mathrm{~ms}$. Therefore, the strain outputs could be obtained from the difference from the first value just before the contact.

Next, the fundamental structure of the sensor should be considered. The advantage of the present sensor compared to the pressure pin-type sensor is that there is no gap between the sensor and the tool. On the contrary, the stress acting on the outside of the sensor may affect the output of the sensor. The force applied outside the detecting surface had no effect on the sensor output in the actual upsetting of the high-temperature cylinder. But, during the process that the outside edge of the deforming material passes through the detecting surface of the sensor, output from the sensor should not be accepted because the detecting surface does not fully contact with the material.

However, there is still room for improvement with this sensor. The size of the sensor could be reduced so that it could be applied to various positions on metal-forming tools. At the moment, the net detecting diameter of the sensor is $4.6 \mathrm{~mm}$ in a block of 8-mm diameter. Even at this size, the sensor can be used during the metal forging of some large product, as shown in Fig. 15. In order to apply the sensor during the cold forging of smaller products, the sensing diameter would need to be smaller. The author intends to develop smaller sensors whose detecting diameter would be $2 \mathrm{~mm}$ in an inserting block $3 \mathrm{~mm}$ in diameter. The accuracy for sensing friction should be examined carefully, and an increase in the sensitivity of the friction measurement will be necessary if plastic deformation under low friction conditions is to be evaluated. Moreover, this sensor only detects friction in one direction in order to detect the frictional stress in any direction, it is essential to consider the design of the two perpendicular beam structures. An image of a sensor which can measure two-dimensional frictions is illustrated in Fig. 16. The sensor comprises two perpendicular detecting beams, and the sensor structure can be fabricated by using electric discharge machining.

This friction sensor will be able to be used for various plastic deformation processes utilizing both hot and cold forging, as well as sheet metal forming, rolling, and extrusion of materials by using various lubrications. Measurements made by this sensor will clearly show boundary stress changes during the plastic deformation process, and the results obtained by it will be useful for setting the boundary condition in numerical analyses. The friction condition measured by this sensor should be compared with the knowledge obtained from various tribology tests, such as ring compression, backward cup extrusion, backward-forward hollow extrusion, spike test, and T-shape compression, in order to investigate friction mechanism during plastic deformation process. Recently, Wang et al. [27] investigated the friction law by using a new tribometer in which a metal sheet specimen with a lot of wedges on the surface was compressed and drawn in a lateral direction. They indicated that there is a constant friction coefficient at low contact pressures and associated constant frictional stress with a high-pressure condition. This information should be investigated with the data measured by this sensor during an actual plastic deformation process.

\section{Conclusions}

A friction sensor for the measurement of pressure and friction in hot forging is developed in this study. To determine the sensor dimensions, the design of the sensor structure is presented. Further, calibration equipment is developed in order to obtain the calibration coefficients of pressure and friction. This equipment is useful to investigate the sensor output before inserting it to the tool surface. The basic measurement results are presented through the compression of a hot steel circular disk and the metal forging by pushing a punch into an axisymmetric material in a semi-closed die by using drop hammer. The applicability of the friction sensor has been discussed.

Acknowledgments The author appreciates Mr. Koji Miyoshi and Tomoki Tate in Komatsu Ltd. in the research cooperation on the experiment in actual hot forging. Additionally, the author gives thanks to the staff in Yamazaki Machinery for accepting the hot forging experiment.

Open Access This article is distributed under the terms of the Creative Commons Attribution 4.0 International License (http:// creativecommons.org/licenses/by/4.0/), which permits unrestricted use, distribution, and reproduction in any medium, provided you give appropriate credit to the original author(s) and the source, provide a link to the Creative Commons license, and indicate if changes were made.

\section{References}

1. Siebel E, Lueg W (1933) Untersuchungen uber die Spannungsverteilung im Walzspalt. Mitt KWI Vol 15:1-14

2. MacGregor, C.W. and Palme, R.B., 1959, The distribution of contact pressure in the rolling of metals, Trans. ASME, J.Basic Eng., 669-680.

3. Matuura Y, Motomura M (1968) Variation of coefficient of friction along the arc of contact, and the length of the arc. J. JSTP 9(86): 173-177

4. Motomura M, Shimamura M (1975) Distributions of rolling pressure along the arc of contact - deformation mechanism during hot rolling I. J. JSTP 16(168):70-77

5. Kato T, Tozawa Y, Nakamura M, Ishikawa T, Inoue K (1980) Development of multi-channel synchronously measuring system of rolling pressure-experimental study on distribution of rolling pressure I. J. JSTP 21(236):800-806 
6. Tozawa Y, Nakamura M, Kato T, Ishikawa T, Inoue K (1980) Condition of measuring the rolling pressure by the pin methodexperimental study on distribution of rolling pressure II. J. JSTP 21(238):999-1005

7. Ishikawa T, Tozawa Y, Nakamura M, Kato T, Kato N (1981) Distribution of rolling pressure measured under various rolling conditions-experimental study on distribution of rolling pressure III. J. JSTP 22(247):816-822

8. Van Rooyen GT, Bachofen WA (1957) Friction in cold rolling, J. Iron steel inst. Vol 186:235-244

9. Matsuura, Y. and Motomura, M., 1968, Distribution of roll pressure over the arc of contact, J. JSTP, Vol.9, no.86, 168-172.

10. Watanabe Y (1969) Influences of strip tensions on the distributions of roll pressure, tangential shear stress and friction coefficient in the roll gap. J. JSTP 10(106):781-792

11. Yoneyama T, Hatamura Y (1987) Development of a die-sensor. JSME Int. 30(262):670-677

12. Yoneyama T, Hatamura Y (1989) Measurement of actual stress and temperature on the roll surface during rolling. JSME Int 32(1):113-117

13. Yoneyama T (1990) Direct measurement of stress and heat between work and tool in metal forming. CIRP Ann 39(1):219-222

14. Jeswiet J, Nyahumwa C (1993) A sensor for measuring metal deformation interface forces, J. Materials Processing Technology 39: 251-268

15. Nyahumwa C (1996) The evolution of a cantilever sensor to measure normal and frictional forces in the roll gap during cold rolling. J. Material Processing Technology 56:463-474

16. Yoneyama T, Hatamura Y, Kitagawa M, Sakakibara K (1994) Development of a pressure sensor using an optical fiber sensor. J. JSTP 35(397):158-163

17. Yoneyama T, Takatsuka K, Kitagawa M, Hatamura Y (1995) Measurement of the contact pressure in aluminum hot extrusion. $\mathrm{J}$ JSTP 36(419):1397-1402
18. Dellah A, Wild PM, Moore TN, Shalaby M, Jeswiet J (2002) An embedded friction sensor based on a strain-gauged diaphragm, journal of manufacturing and engineering. Transactions of the ASME 124:523-527

19. Lupoi R, Osman FH (2005) Under surface pressure sensing technique for the evaluation of contact stresses. J Mater Process Technol 164-165:1537-1543

20. Jesweit J, Arentoft M, Henningsen P (2006) Methods and devices used to measure friction in rolling. I Mech E Proc Part B: J Engineering Manufacture 220:49-57

21. Yoneyama T (1999) Development of a new pressure sensor and its application to the measurement of contacting stress in extrusion. J Mater Process Technol 95:71-77

22. Yoneyama T, Takahashi M (2004) Development of a friction sensor and measurement in aluminum hot extrusion. J. JSTP 45(521):82-86

23. Yoneyama T, Takahashi M (2006) Pressure and friction between billet and container in aluminum hot extrusion. J. JSTP 47(545): 527-531

24. Makino T, Michimoto T, Yogo Y (2014) Development of direct measurement method for friction force at tool surface, the proceedings of the 65th. Japanese Joint Conference for the Technology of Plasticity $175-176$

25. Zhang Q, Felder E, Bruschi S (2009) Evaluation of friction condition in cold forging by using T-shape compression test. J Mater Process Technol 209:5720-5729

26. Shimoda, K. and Yoneyama, T., 2012, Application of friction and pressure sensor to hot forging, The proceeding of the 2012 Japanese spring conference for the technology of plasticity, 147-148.

27. Wang ZG, Yoshikawa Y, Suzuki T, Osakada K (2014) Determination of friction law in dry metal forming with DLC coated tool. CIRP Annals-Manufacturing Technology 63:277-280 